\title{
Hymenoptera "Parasitica" in the state of Mato Grosso do Sul, Brazil
}

\author{
Eduardo Mitio Shimbori', Helena Carolina Onody', Daniell Rodrigo Rodrigues Fernandes², Rogério \\ Silvestre ${ }^{3}$, Marcelo Teixeira Tavares ${ }^{4} \&$ Angélica Maria Penteado-Dias ${ }^{1}$
}

\footnotetext{
1. Departamento de Ecologia e Biologia Evolutiva, Universidade Federal de São Carlos, São Carlos, SP, Brazil. (shimbori@gmail.com)

2. Programa de Pós-Graduação em Entomologia, Instituto Nacional de Pesquisas da Amazônia INPA, Manaus, AM, Brazil.

3. Faculdade de Ciências Biológicas e Ambientais da Universidade Federal da Grande Dourados, Dourados, MS, Brazil.

4. Departamento de Ciências Biológicas, Universidade Federal do Espírito Santo, Vitória, ES, Brazil.
}

Received 22 November 2016

Accepted 6 February 2017

DOI: $10.1590 / 1678-4766 e 2017121$

ABSTRACT. A checklist composed of 105 species of parasitic Hymenoptera, which includes the non-aculeate Apocrita, recorded in the state of Mato Grosso do Sul (MS), Brazil, is presented. A new list, containing 153 genera obtained in recent surveys is also presented; out of these 131 are new records. The major knowledge gaps for these organisms in the State and the prospects for future studies for these organisms are discussed.

KEYWORDS. Parasitoids, species checklist, distribution, biodiversity, new records, Biota-MS Program.

RESUMO. Hymenoptera "Parasitica" no Estado do Mato Grosso do Sul, Brasil. Apresentamos o checklist com 105 espécies de Hymenoptera "Parasitica", que inclui os Apocrita não aculeados, registradas no estado de Mato Grosso do Sul (MS) e uma lista inédita com 153 gêneros obtidos em levantamentos recentes, dos quais 131 são novos registros. As principais lacunas do conhecimento para estes organismos no estado e as perspectivas para estudos futuros são discutidas.

PALAVRAS-CHAVE. Parasitoides, lista de espécies, distribuição, biodiversidade, novos registros, Programa Biota-MS

It is widely recognized, among the researchers of the area of invertebrates, that there is a lack of studies for the area in the Center-West region of Brazil, mainly in the state of Mato Grosso do Sul (Lewinsohn \& Prado, 2002). LEWINSOHN et al. (2005) pointed out the wide scientific knowledge gap that exists about biological diversity of biomes in Brazil. According to them, the Amazonian and Atlantic Forests are the best studied ones, whereas the Pantanal and Caatinga are the least known, as seen on recent inventories of species. BRANDÃO et al. (2000) reported that after the Northeast region, the Center-West region is classified as the second least known for the invertebrates. In the same study, the authors considered as inexistent the knowledge and collection status for parasitoid wasps (Hymenoptera), represented by the Ichneumonoidea, in the Pantanal area, a biome whose largest portion is in the state of Mato Grosso do Sul (MS).

The difficult access to the region in the past, as well as the low demographic rate and conflicts for land in the area are among the reasons that explain the gaps of knowledge in the region, facts that limited the visit of naturalists, mainly during the period of large expeditions which were common until the middle of the $20^{\text {th }}$ century. The entomological knowledge in Brazil was built based on specimens collected by those expeditions and by naturalists from Europe and USA, who concentrated their efforts in the exuberant wet forests of this country, such as the Atlantic and Amazon Rainforests. Henry W. Bates in the state of Amazonas and Fritz Plaumann in the state of Santa Catarina, are two well-known examples of this phase. The greatest efforts in sampling the fauna of the Center-West region occurred during the last years of the $19^{\text {th }}$ century, and were carried out by Herbert $\mathrm{H}$. Smith, a naturalist from the USA, who spent four years in the state of Mato Grosso (Smith, 1922; KunZler et al., 2011). Apart from the geological work he performed, Herbert Smith collected vertebrates, insects and fossils. Although he concentrated his studies in the Chapada dos Guimarães, he also carried out collections in other regions, including Corumbá (MS). During the $20^{\text {th }}$ century, a relevant expedition in the region was the "Royal Geographical Society" in Nova Xavantina, state of Mato Grosso, which brought a large contribution for the knowledge of Hymenoptera, in particular eusocial wasps (RICHARDS, 1978).

With the establishment of research centers in the country, the Brazilian Entomology initiated a new phase. However, the concentration of these research centers in the South, Southeast and North regions were determinant for the lack of studies about the Hymenoptera parasitoids fauna in the Center-West region. Within this new context, the situation of the state of Mato Grosso do Sul was aggravated by its relative recent creation. 
The Hymenoptera contain approximately 115,000 described species, being the third Order in number of species after Coleoptera and Lepidoptera. When considering the nondescribed species, it is possible that the Hymenoptera be the richest order in number of species (GASTON, 1991; STORK, 1991; Grissell, 1999). GASTON et al. (1996) estimated that the number of species could reach as much as three million. More conservative estimates forecast a number of species ranging between 600,000 and 1,200,000 (GRIMALDI \& ENGEL, 2005).

The traditional division of the Hymenoptera order into two suborders, Symphyta and Apocrita, is recognized to be artificial, since the former group composes an ancestral grade to the Apocrita + Orussoidea (SHARKEY et al., 2012). The latter compose a clade that theoretically has a common ancestral of ectoparasitoid habit, from which originated the large diversity of habits currently known to be present on the Apocrita, such as endoparasitism, cecidogenesis, predation and eusocial behavior (WhitFIELD, 1992, 2003).

The Apocrita form a monophyletic group, which is also traditionally divided into two groups, the Aculeata (monophyletic) and the parasitoid wasps, also known as "Parasitica". A large proportion of phylogenetic studies point "Parasitica" as a paraphyletic group, being Aculeata a sister group of Evanioidea (SHARKEY et al., 2012). Despite of the predominance of the parasitoid habit found in the non-aculeate Apocrita, some of the evolutionary lineages developed different habits, mainly the associations as and galls formations (WHITFIELD, 2003; ZALDÍVAR-RIVERóN et al., 2007).

Among the Apocrita, Aculeata and parasitoids have approximately the same number of described species. A large proportion of the non-described species, however, is of Hymenoptera parasitoids (SHARKEY, 2007). In the Neotropical region 24,000 species of Hymenoptera are known, out of which little less than half are of non-aculeate species (FernándeZ \& Sharkey, 2006). According to FernándeZ (2006), there is at least 80,000 species in the Neotropical region, although there is not an estimate account for the region.

From the 89 families of Hymenoptera recognized by SHARKEY (2007), 76 occur in the Neotropical region, out of which 41 are within the "Parasitica" series (i.e. Apocrita, except Aculeata) (FERNÁNDEZ, 2006; SHARKEY, 2007). Among the groups with the poorest degree of knowledge for the Neotropical region are the Ceraphronoidea, Cynipoidea, Evanioidea, Platygastroidea and Chalcidoidea. Considering the number of species, the Ichneumonoidea can also be included within those groups (FERnÁNDEZ \& SHARKEY, 2006).

This work analyzed all families belonging to the "Parasitica" series, which includes all Apocrita but Aculeata ones (Chrysidoidea, Vespoidea and Apoidea). A checklist is presented for the species recorded for the state, since most consulted catalogues do not consider, or were elaborated, before the creation of the state of Mato Grosso do Sul (MS). Also, an unprecedented list, containing genera obtained through recent surveys is presented.

\section{MATERIAL AND METHODS}

The composition of the list of species was carried out based on bibliographic material. As a starting point, available catalogues for families and superfamilies were used. The groups of parasitoid wasps with available catalogues are: Chalcidoidea (NOYES, 2013), Ichneumonoidea (Yu et $a l ., 2012)$, Ichneumonidae (Townes \& Townes, 1966), Braconidae (SHENEFELT parts 1 to 16, published between 1965 and 1980), "Parasitica" (for Brazil) (De SANTIS, 1980), Platygastridae (VLUG, 1995), Proctotrupoidea sensu lato (Jonhson, 1992), Aulacidae (Smith, 2001), Stephanidae (Aguiar, 2004) and Trigonalidae (Weinstein \& Austin, 1991).

The locality of the occurrences of all species recorded for the state of Mato Grosso were designated, through the consultation to original descriptions and recent publications, in order to adequate its occurrence to the Brazilian current political division. The toponymies referring to localities within the current state of Mato Grosso do Sul are listed and the occurrences of species in those localities transferred.

A newly elaborated list of genera identified for the state was produced. The material examined was mainly collected in Pantanal area within the municipality of Corumbá, MS. The collection of this material was carried out under the Biota-MS Program (FAPESP 2010/52314-0) using Malaise traps. Material collected in Cerrado area within the municipality of Campo Grande (MS) was also identified. Also, material collected using Malaise traps by students from the Biological Sciences course at Universidade Católica Dom Bosco, under the supervision of Dr. Antonia Railda Roel, was identified. Additional material, deposited in the Coleção de Hymenoptera do Museu de Biodiversidade da Faculdade de Ciências Biológicas e Ambientais/Universidade Federal da Grande Dourados (MuBio-Hym), is also included in the list, only for the Braconidae. This material was collected with Möericke traps in the Parque Nacional da Serra da Bodoquena and in the Municipality of Porto Murtinho, both in the state of Mato Grosso do Sul (MS). In addition to the MuBio-Hym's collection, the collected material is also deposited at the Coleção Entomológica do Museu de Zoologia da USP (MZUSP), at the Coleção Entomológica do Depto. de Ecologia e Biologia Evolutiva da UFSCar (DCBU) and at the Coleção de Invertebrados da Universidade Católica Dom Bosco (UCDB).

The identification was carried out based on current literature and keys for each family in addition to consultations with specialists. The identification in genus level is justified by the high diversity of the group and the lack of revisions that enable a reliable identification at the species level.

\section{RESULTS AND DISCUSSION}

List of species. The list of species presented here includes only species with existing records in the literature which, given the precise information about the locality of their collection could be confirmed as belonging to the state 
of Mato Grosso do Sul. As much as 139 records for 105 species, belonging to 63 genera in 18 of the 41 known families of "Parasitica" Hymenoptera (Tab. I), were accounted for. This information was obtained from 51 references published between 1904 and 2013. In addition to information taken from the literature, six species of Ichneumonidae are being recorded for the first time in this work.

Taxonomic works are 22 and the remaining 29 were concerned with insects' natural enemies, mostly of economic relevance. The papers on natural enemies were published after the state of Mato Grosso do Sul was established; from all the taxonomic works available, only four were published before this date (Fig. 1). Ashmead (1904), for instance, contributed with 39 records of species of Chalcidoidea from a total of 59 records of this superfamily for the state of Mato Grosso do Sul. This valuable contribution was carried out based on specimens collected by Herbert Smith, most of them in Corumbá (MS) (see Fig. 2). Taxonomic works present more species number as expected, but works on natural enemies contain valuable information about the host-parasitoid association, useful not only for biological control programs, also for a better understanding of their interaction patterns at the ecological and evolutionary scale.

The checklist comprises a majority of species with previous record for the state of Mato Grosso, transferred here for the state of Mato Grosso do Sul. Hence, the number of species presented in this list exceeds the sum of the numbers in previous catalogs for this state. However, this number can be at least as three times as bigger considering the number of genera identified in the present study.

In order to avoid possible misunderstandings between the two states, the species with records of occurrence in both states are indicated in Table I. Therefore, all species without indication do not have records for the state of Mato Grosso. Records in other states and countries were not considered.

List of genera obtained from recent surveys. A total of 153 genera of "Parasitica" Hymenoptera were identified, out of which 131 (85\%) are new records (Tab. II). These genera belong to 20 families, out of which seven are recorded for the first time in the state of Mato Grosso do Sul. The Ceraphronoidea is recorded here for the first time. Most of the identified material was collected in Pantanal area at the municipality of Corumbá.

The families Aulacidae, Diapriidae, Stephanidae and Trichogrammatidae were not found in the examined material. Platygastroidea was relatively commonly collected, although the family was not identified because the lack of keys to genera and specialists in Brazil. Therefore it is not present in the genera list.

Main research groups. The main research groups in Hymenoptera parasitoids are concentrated in the Southeast region. A list of these groups, together with their researcher leaders follows: (1) Alexandre Pires Aguiar, Universidade Federal do Espírito Santo (UFES), Vitória, ES; groups of interest: Stephanoidea and Cryptinae (Ichneumonidae); (2) Alice Fumi Kumagai, Universidade Federal de Minas Gerais, Belo Horizonte, MG; group of interest: Ichneumonidae
(Pimplinae); (3) Angélica Maria Penteado-Dias, Universidade Federal de São Carlos (UFSCar), São Carlos, SP; group of interest: Ichneumonoidea; (4) Ayres de Oliveira Menezes Júnior, Universidade Estadual de Londrina, Londrina, PR; group of interest: Chalcidoidea (Encyrtidae and Leucospidae); (5) Celso Oliveira Azevedo, Universidade Federal do Espírito Santo (UFES), Vitória, ES; group of interest: Bethylidae; (6) Jorge Anderson Guimarães, Embrapa Hortaliças, Brasília, DF; group of interest: Figitidae (Eucoilinae); (7) Marcelo Teixeira Tavares, Universidade Federal do Espírito Santo (UFES), Vitória, ES; group of interest: Chalcididae; (8) Nelson Wanderley Perioto, APTA Ribeirão Preto, Ribeirão Preto, SP; group of interest: Chalcidoidea (Eurytomidae, Eulophidae and Tanaostigmatidae); (9) Ranyse Barbosa Querino da Silva, Embrapa Meio-Norte, Teresina, PI; group of interest: Chalcidoidea (Trichogrammatidae); (10) Roberto Antonio Zucchi, Escola Superior de Agricultura Luiz de Queiroz. Piracicaba, SP; group of interest: Trichogrammatidae; (11) Valmir Antonio Costa, Instituto Biológico, Campinas, SP; group of interest: Chalcidoidea (Eulophidae).

There are not yet research groups in taxonomy and systematic of parasitic Hymenoptera in Mato Grosso do Sul. Regarding the inventory of the Hymenoptera parasitoids in MS, only one M.Sc. dissertation was found, produced by Vander Carbonari under the Graduate Program in Entomology and Biology Conservation at UFGD (Programa de Pós-Graduação em Entomologia e Conservação da Biodiversidade, Universidade Federal da Grande Dourados). Carbonari carried out collections during two years in the Serra da Bodoquena (MS) collecting 1,243 specimens of the Hymenoptera parasitoids; however, the deposited material in the MuBio-UFGD's collection is identified only at the family level (CARBONARI, 2009). Under-graduate and Graduate students at the Universidade Católica Dom Bosco (UCDB) have been carrying out a survey of the entomofauna in the region of Campo Grande (MS), under the supervision of Dr. Antonia Railda Roel with the collaboration of the research group headed by Dr. Angélica Maria Penteado-Dias. Those students are being trained in the collection, preservation and identification of Hymenoptera, mainly the parasitoid ones.

Publications, locally produced, containing records of parasitoid Hymenoptera species were carried out by Dr. Manoel Araécio Uchôa-Fernandes research group at UFGD, in Dourados (MS), whose main group of interest are the fruit flies (Diptera: Tephritoidea) and their interaction with eventual parasitoids. More recently, the research groups leaded by Dr. Fabrício Fagundes Pereira from UFGD and by Dr. Harley Nonato de Oliveira from Embrapa Agropecuária Oeste, both in Dourados (MS), are researching biological aspects of parasitoid species with a focus on their use as biological controllers.

Main holdings. As happens with the research groups, the main holdings in Brazil are mostly concentrated in the Southeast region of the country, such as: DCBU, São Carlos (SP); MZUSP, São Paulo (SP); UFES, Vitória (ES); FIOC, Rio de Janeiro (RJ); MNRJ, Rio de Janeiro (RJ). In the South region, the DZUP and DCMP are both in Curitiba (PR); and 
Tab. I. Checklist of Hymenoptera "Parasitica" species recorded in Mato Grosso do Sul State (MS), Brazil (1, species recorded in Mato Grosso and Mato Grosso do Sul States; 2, new record locality; 3, record in distribution map, municipality/locality not provided).

\begin{tabular}{|c|c|c|}
\hline Species & Municipalities/localities* in MS & References \\
\hline \multicolumn{3}{|l|}{ CHALCIDOIDEA } \\
\hline \multicolumn{3}{|l|}{ Aphelinidae } \\
\hline Encarsia porteri (Mercet, 1928) & Campo Grande & ANDRADE-FILHO et al., 2012 \\
\hline \multicolumn{3}{|l|}{$\begin{array}{l}\text { Chalcididae } \\
\text { S }\end{array}$} \\
\hline Brachymeria flavopicta (Ashmead, 1904) & Corumbá & ASHMEAD, 1904 \\
\hline Brachymeria implexa (Walker, 1862) & Corumbá & ASHMEAD, 1904 \\
\hline Brachymeria ovata (Say, 1824) & Ribas do Rio Pardo & Berti-Filho et al., 1980 \\
\hline Ceyxia flaviscapus Girault, 1911 & Corumbá & ANDRADE \& TAVARES, 2009 \\
\hline Ceyxia concitator (Walker, 1862) & Corumbá & ANDRADE \& TAVARES, 2009 \\
\hline Ceyxia diminuta Andrade \& Tavares, 2009 & Corumbá & ANDRADE \& TAVARES, 2009 \\
\hline Ceyxia ventrispinosa Girault, 1911 & Corumbá & ANDRADE \& TAVARES, 2009 \\
\hline Conura acuminata (Ashmead, 1904) & Corumbá & AsHMEAD, 1904 \\
\hline Conura acuta (Fabricius, 1804) ${ }^{1}$ & Corumbá & ASHMEAD, 1904 \\
\hline Conura albomaculata (Ashmead, 1904) ${ }^{1}$ & Corumbá & ASHMEAD, 1904 \\
\hline Conura brancensis (Ashmead, 1904) & Itaporã & ASHMEAD, 1904 \\
\hline Conura celsa (Walker, 1864) & Corumbá & ASHMEAD, 1904 \\
\hline Conura corumbensis (Ashmead, 1904) & Corumbá & ASHMEAD, 1904 \\
\hline Conura costalimai (De Santis, 1980) & Corumbá & AshmeAD, 1904; De SANTIS, 1980 \\
\hline Conura flava (Fabricius, 1804) ${ }^{1}$ & Corumbá & ASHMEAD, 1904 \\
\hline Conura fusiformis (Ashmead, 1904) & Itaporã & ASHMEAD, 1904 \\
\hline Conura lineocoxalis (Ashmead, 1904) & Corumbá & ASHMEAD, 1904 \\
\hline Conura media (Ashmead, 1904) & Corumbá & ASHMEAD, 1904 \\
\hline Conura mourei (De Santis, 1980) & Corumbá & Ashmead, 1904; De SANTIS, 1980 \\
\hline Conura quadripunctata (Fabricius, 1804) & Corumbá, Itaporã & ASHMEAD, 1904 \\
\hline Conura timida (Ashmead, 1904) & Corumbá & ASHMEAD, 1904 \\
\hline Corumbichalcis corumbicola (Ashmead, 1904) & Corumbá & Ashmead, 1904; Delvare, 1992 \\
\hline \multicolumn{3}{|l|}{ Encyrtidae } \\
\hline Aenasius tachigaliae (Brues, 1922) & Corumbá & MERCET, 1926; COMPERE, 1937 \\
\hline Hexacladia smithii Ashmead, 1891 & $\begin{array}{l}\text { Dourados, Ponta Porã, } \\
\text { Itaporã, Caarapó }\end{array}$ & Godoy et al., 2007; 2010 \\
\hline \multicolumn{3}{|l|}{ Eucharitidae } \\
\hline Orasema rapo (Walker, 1839) & Corumbá & ASHMEAD, 1904 \\
\hline \multicolumn{3}{|l|}{ Eulophidae } \\
\hline Alveoplectrus corumbae (Ashmead, 1904) & Corumbá & ASHMEAD, 1904 \\
\hline Aprostocetus diplodisis Crawford, 1907 & Selvíria & CAMPOS et al., 1998 \\
\hline Euderus brasiliensis (Ashmead, 1904) ${ }^{1}$ & Corumbá & ASHMEAD, 1904 \\
\hline Euplectrus brasiliensis Ashmead, 1904 & Itaporã & ASHMEAD, 1904 \\
\hline Euplectrus floryae Schauff, 2001 & Dourados & BELLON et al., 2013 \\
\hline Horismenus corumbae Ashmead, 1904 & Corumbá & ASHMEAD, 1904 \\
\hline Sympiesis obscura (Ashmead, 1904) & Corumbá & ASHMEAD, 1904 \\
\hline Tetrastichus howardi (Olliff, 1893) & Dourados & VARGAS et al., 2011 \\
\hline \multicolumn{3}{|l|}{ Eupelmidae } \\
\hline Anastatus auriceps Ashmead, $1904^{1}$ & Corumbá & ASHMEAD, 1904 \\
\hline Brasema corumbae (Ashmead, 1904) & Corumbá & ASHMEAD, 1904 \\
\hline Eupelmus compressiventris Ashmead, 1904 & Corumbá & ASHMEAD, 1904 \\
\hline Eupelmus persimilis Ashmead, 1904 & Corumbá & ASHMEAD, 1904 \\
\hline \multicolumn{3}{|l|}{ Eurytomidae } \\
\hline Axima brevicornis Ashmead, $1904^{1}$ & Corumbá & ASHMEAD, 1904 \\
\hline Isosomodes gigantea (Ashmead, 1886) & Corumbá & ASHMEAD, 1904 \\
\hline \multicolumn{3}{|l|}{ Mymaridae } \\
\hline Anagrus urichi Pickles, 1932 & Campo Grande & VALÉRIO \& OLIVEIRA, 2005 \\
\hline \multicolumn{3}{|l|}{ Pteromalidae } \\
\hline Chalcedectus annulipes Ashmead, $1904^{1}$ & Corumbá & ASHMEAD, 1904 \\
\hline Lelaps abdominalis Ashmead, 1904 & Itaporã & AsHMEAD, 1904 \\
\hline Leptofoenus westwoodi (Ashmead, 1895) 1 & Itaporã, Campo Grande ${ }^{2}$ & AsHMEAD, 1904 \\
\hline Lycisca ignicaudata Westwood, $1874^{1}$ & Corumbá & ASHMEAD, 1904 \\
\hline Psilocera brasiliensis (Ashmead, 1904) & Corumbá & ASHMEAD, 1904 \\
\hline Psilocera clavicornis (Ashmead, 1904) & Corumbá & ASHMEAD, 1904 \\
\hline Spalangia endius Walker, 1839 & $\begin{array}{l}\text { Anastácio, Terenos, Aquidauana, } \\
\text { Rochedo, Corumbá, Parnaíba }\end{array}$ & $\begin{array}{l}\text { Silveira et al., 1989; Sereno, 2000; Sereno \& } \\
\text { Sereno, 2000; UchôA-FernandeS } \text { et al., } 2003\end{array}$ \\
\hline Spalangia gemina Boucek, 1963 & $\begin{array}{l}\text { Anastácio, Terenos, } \\
\text { Aquidauana, Rochedo }\end{array}$ & UCHÔA-FERNANDES et al., 2003 \\
\hline Spalangia nigroaenea Curtis, 1839 & Corumbá, Parnaíba & $\begin{array}{l}\text { SiLVEIRA et al., 1989; SERENo 2000; } \\
\text { SERENO \& SERENO, } 2000\end{array}$ \\
\hline Spalangia plaumanni Gibson, 2009 & Rio Caraguatá* $\left(21^{\circ} 48^{\prime} \mathrm{S}, 52^{\circ} 27^{\prime} \mathrm{W}\right)$ & GIBSON, 2009 \\
\hline
\end{tabular}


Tab. I. Cont.

\begin{tabular}{|c|c|c|}
\hline Species & Municipalities/localities* in MS & References \\
\hline \multicolumn{3}{|l|}{ Torymidae } \\
\hline Plesiostigmodes brasiliensis Ashmead, 1904 & Corumbá & ASHMEAD, 1904 \\
\hline Torymus aprilis (Ashmead, 1904) & Corumbá & ASHMEAD, 1904 \\
\hline Torymus holcaspoideus (Ashmead, 1904) ${ }^{1}$ & Corumbá & ASHMEAD, 1904 \\
\hline Torymus nonacris (Walker, 1842) & Corumbá & ASHMEAD, 1904 \\
\hline \multicolumn{3}{|l|}{ Trichogrammatidae } \\
\hline Trichogramma galloi Zucchi, 1988 & Brasilândia & $\begin{array}{l}\text { ZuCChi \& Monteiro, 1995; } \\
\text { BOtelho et al., } 1999\end{array}$ \\
\hline $\begin{array}{l}\text { Trichogramma manicobai Brun, Moares \& Soares, } \\
1984\end{array}$ & Ivinhema & OliveIRA et al., 2010; GoMEZ et al., 2006 \\
\hline $\begin{array}{l}\text { Trichogramma marandobai Brun, Moraes \& Soares, } \\
1986\end{array}$ & Dourados, Itaquiraí, Ivinhema & OLIVEIRA et al., 2010; GoMEZ et al., 2006 \\
\hline Trichogramma pretiosum Riley, 1879 & $\begin{array}{l}\text { Dourados, Glória de Dourados, } \\
\text { Ivinhema }\end{array}$ & $\begin{array}{l}\text { FERnÁNDEZ et al., 1999; Oliveira et al., 2010; } \\
\text { GoMEZ et al., } 2006\end{array}$ \\
\hline
\end{tabular}

\section{CYNIPOIDEA}

Figitidae

Aganaspis nordlanderi Wharton, 1998

Ganaspis fulvocincta Kieffer, 1909

Kleidotoma nigra (Hartig, 1840)

Lopheucoila anastrephae (Rhower, 1919)

Neralsia fossulata (Kieffer, 1909)

Odontosema anastrephae (Borgmeier, 1935)

Paraganaspis egeria Díaz \& Gallardo, 1996

Triplasta atrocoxalis (Ashmead, 1895)

Trybliographa infuscata Gallardo, Díaz \& Uchôa-

Fernandes, 2000

DIAPRIOIDEA

Diapriidae

Trichopria haematobiae (Ashmead, 1893)

EVANIOIDEA

Aulacidae

Pristaulacus petiense Smith, 2010

ICHNEUMONOIDEA

Braconidae

Aivalykus flavus (Marsh, 1993)

Alabagrus mojos Sharkey, $1988^{1}$

Alabagrus nigritulus Szepligeti, 1902

Aleiodes melanopterus (Erichson, 1848) ${ }^{1}$

Aphidius uzbekistanicus Luzhetzki, 1960

Asobara anastrephae (Muesebek, 1958)

Bohartiellus cornutus Marsh,1983

Cotesia flavipes (Cameron, 1891)

Diaeretiella rapae (McIntosh, 1855)

Doryctobracon areolatus (Szépligeti, 1911)

Doryctobracon fluminensis (Costa Lima, 1937)

Doryctopambolus pilcomayensis (Achterberg \&

Braet, 2004)

Exasticolus fuscicornis (Cameron, 1887)

Gnathopleura astarte (Haliday, 1838) ${ }^{1}$

Gnathopleura semirufa (Brullé, 1846) ${ }^{1}$

Opius anastrephae Viereck, 1913

Opius bellus Gahan, 1930

Pharpa dubiosum (Szépligeti,1914)

Praon gallicum Starý, 1971

Rinamba platyfemur (Marsh,1993)

Sharkeyella pilosus Marsh, 1993

Verae peculya Marsh, 1993

Ichneumonidae

Digonocryptus diversicolor (Viereck, 1913)
Anastácio, Terenos, Aquidauana,

Rochedo

Selvíria

Selvíria

Anastácio, Terenos, Aquidauana,

Rochedo

Selvíria

Anastácio, Terenos, Aquidauana,

Rochedo

Selvíria

Selvíria

Anastácio, Terenos, Aquidauana,

Rochedo

Corumbá

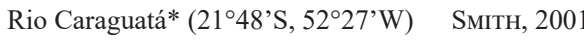

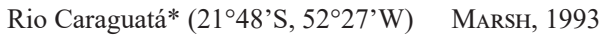

Dourados (Itaum)

MS (map) ${ }^{3}$

Campo Grande, Dourados, Serra da

Bodoquena $^{2}$

Dourados

Anastácio, Terenos, Aquidauana,

Rochedo

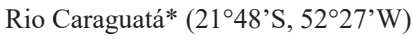

Brasilândia,

Dourados

Anastácio, Terenos, Aquidauana,

Rochedo

Anastácio, Terenos, Aquidauana,

Rochedo

Bodoquena

Dourados, Itaporã

Dourados

Campo Grande, Corumbá

Anastácio, Aquidauana, Miranda,

Terenos, Rochedo

Anastácio, Terenos, Aquidauana,

Rochedo

MS (map) ${ }^{3}$

Dourados

Rio Caraguatá*

Rio Caraguatá* $\left(21^{\circ} 48^{\prime} \mathrm{S}, 52^{\circ} 27^{\prime} \mathrm{W}\right)$

Rio Caraguatá* $\left(21^{\circ} 48^{\prime} \mathrm{S}, 52^{\circ} 27^{\prime} \mathrm{W}\right)$

Dourados (Itaum)
UChÔA-FERnANDES et al., 2003a, 2003b

Díaz \& Gallardo, 1996; Díaz et al., 2000

Díaz \& Gallardo, 1996; Díaz et al., 2000

GuIMARÃES et al., 1999;

UChÔA-FERnANDES et al., 2003b

Díaz \& Gallardo, 1996; Díaz et al., 2000

GUIMARÃES et al., 1999;

UCHÔA-FERNANDES et al., 2003 a, 2003b

Díaz \& GaLlardo, 1996; DíAz et al., 2000

Díaz \& Gallardo, 1996; Díaz et al., 2000

GALLARDO et al., 2000;

UCHÔA-FERNANDES et al., 2003a, 2003b

Sereno, 2000; Sereno \& Sereno, 2000

SHARKEY, 1988

SHARKEY, 1988

Shimbori \& Penteado-Dias, 2011

Gomez \& Rumiatto, 1989; StaRÝ et al., 2007

UCHÔA-FERNANDES et al., 2003a,b

MARSH, 1983

BoteLHo et al., 1999

Mussury \& Fernandes, 2002;

GOMEZ \& RumiatTo, 1989; StaRÝ et al., 2007

UCHÔA-FERNANDES et al., 2003

UCHÔA-FERNANDES et al., 2003

NuNES et al., 2012

FigUeIREDo et al., 2006

Arouca \& Penteado Dias, 2009

Arouca \& Penteado Dias, 2009

UCHÔA-FERNANDES et al., 2003;

CAIRES et al., 2009

UCHÔA-FERNANDES et al., 2003a,b

SHARKEY, 1986

Gomez \& RumiatTo, 1989; StaRÝ et al., 2007

BRAET \& VAN ACHTERBERG, 2001

Marsh, 1993

MARSH, 1993

Aguiar \& Ramos, 2011 
Tab. I. Cont.

\begin{tabular}{|c|c|c|}
\hline Species & Municipalities/localities* in MS & References \\
\hline Eiphosoma dentator (Fabricius, 1804) & Bodoquena, Corumbá ${ }^{2}$ & Costa Lima, 1953 \\
\hline Eiphosoma laphygmae Costa Lima, 1953 & Corumbá $^{2}$ & First record \\
\hline Eiphosoma macrum (Enderlein, 1921) & Corumbá ${ }^{2}$ & First record \\
\hline Eiphosoma matogrossense Costa Lima, 1953 & Salobra & Costa Lima, 1953 \\
\hline Eiphosoma nigrovittatum Cresson, 1865 & Corumbá ${ }^{2}$ & First record \\
\hline Enicospilus flavoscutellatus (Brullé, 1846) & Corumbá ${ }^{2}$ & First record \\
\hline Enicospilus glabratus (Morley, 1913) & Campo Grande ${ }^{2}$ & First record \\
\hline Ophion flavidus Brullé, 1846 & Rio Brilhante ${ }^{2}$ & First record \\
\hline \multicolumn{3}{|l|}{ PLATYGASTROIDEA } \\
\hline \multicolumn{3}{|l|}{ Platygastridae } \\
\hline Telenomus pachycoris (Costa Lima, 1928) & Dourados & OliveIra \& SiLVa, 2011 \\
\hline Telenomus podisi (Ashmead, 1893) & $\begin{array}{l}\text { Dourados, Caarapó, Rio Brilhante, } \\
\text { Ponta Porã, São Gabriel do Oeste, } \\
\text { Itaporã }\end{array}$ & $\begin{array}{l}\text { Godoy \& Ávila, 2000; Godoy et al., 2005, } 2007 \\
\text { CARVALHO, 2007; ArCe, } 2007\end{array}$ \\
\hline Trissolcus basalis (Wollaston, 1858) & São Gabriel do Oeste & GoDoy et al., 2005 \\
\hline \multicolumn{3}{|l|}{ STEPHANOIDEA } \\
\hline \multicolumn{3}{|l|}{ Stephanidae } \\
\hline Aguiarina adustus (Aguiar, 1998) & Rio Caraguatá* $\left(21^{\circ} 48^{\prime} \mathrm{S}, 52^{\circ} 27^{\prime} \mathrm{W}\right)$ & AGUIAR, 1998; 2004 \\
\hline
\end{tabular}

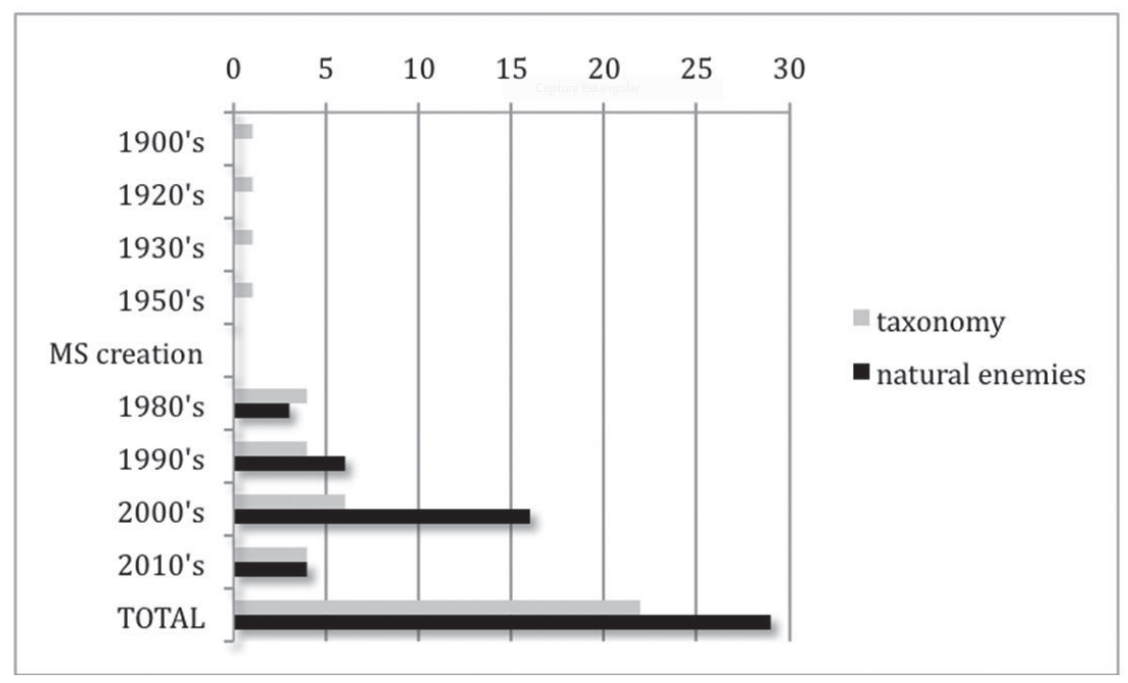

Fig. 1. Number of publications with Hymenoptera "Parasitica" species recorded, grouped by decade. Works are classified according to its subject: taxonomy or natural enemies (see text).

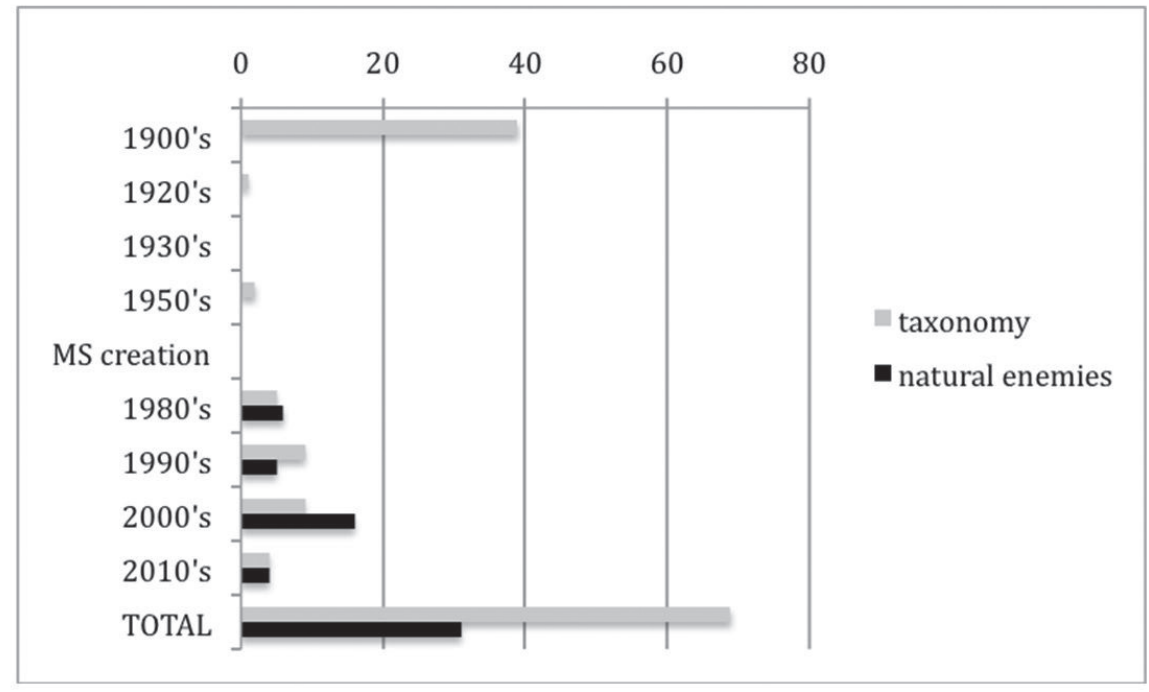

Fig. 2. Number of newly recorded Hymenoptera "Parasitica" species grouped by decade. Works are classified according to its subject: taxonomy or natural enemies (see text). 
Tab. II. List of Hymenoptera "Parasitica" genera identified from recent surveys in the state of Mato Grosso do Sul (MS), Brazil (*, new record for taxon in Mato Grosso do Sul State).

\begin{tabular}{|c|c|}
\hline Taxa & Locality in MS \\
\hline \multicolumn{2}{|l|}{ CERAPHRONOIDEA* } \\
\hline \multicolumn{2}{|l|}{ Ceraphronidae* } \\
\hline Aphanogmus Thomson, 1853* & Corumbá \\
\hline \multicolumn{2}{|l|}{ CHALCIDOIDEA } \\
\hline \multicolumn{2}{|l|}{ Chalcididae } \\
\hline Aspirrhina Kirby, 1883* & Corumbá \\
\hline Brachymeria Westwood, 1829 & Corumbá \\
\hline Conura Spinola, 1837 & Corumbá \\
\hline Dirhinus Dalman, 1818* & Corumbá \\
\hline Haltichella Spinola, $1811 *$ & Corumbá \\
\hline Hockeria Walker, 1834* & Corumbá \\
\hline Melanosmicra Ashmead, 1904* & Corumbá \\
\hline Notaspidium Dalla Torre, $1897 *$ & Campo Grande, Corumbá \\
\hline Stypiura Kirby, $1883^{*}$ & Corumbá \\
\hline \multicolumn{2}{|l|}{ Encyrtidae } \\
\hline Aenasius Walker, 1846 & Corumbá \\
\hline Anagyrus Howard, 1896* & Corumbá \\
\hline Blepyrus Howard, 1898* & Corumbá \\
\hline Encyrtus Latreille, 1809* & Corumbá \\
\hline Gahaniella Timberlake, $1926^{*}$ & Corumbá \\
\hline Hemencyrtus Ashmead, 1900* & Corumbá \\
\hline Leptomastix Föerster, 1856* & Corumbá \\
\hline Metaphycus Mercet, 1917* & Corumbá \\
\hline Syrphophagus Ashmead, 1900* & Corumbá \\
\hline \multicolumn{2}{|l|}{ Eucharitidae } \\
\hline Kapala Cameron, 1884* & Corumbá \\
\hline Lophyrocera Cameron, 1884* & Campo Grande \\
\hline Obeza Heraty, $1985^{*}$ & Corumbá \\
\hline Pseudochalcura Ashmead, 1894* & Corumbá \\
\hline \multicolumn{2}{|l|}{ Eulophidae } \\
\hline Chrysocharis Föerster, 1856* & Corumbá \\
\hline Closterocerus Hartig, 1847* & Corumbá \\
\hline Elasmus Westwood, 1833* & Corumbá \\
\hline Euplectrus Westwood, 1832 & Corumbá \\
\hline Galeopsomyia Girault, 1916* & Corumbá \\
\hline \multicolumn{2}{|l|}{ Eupelmidae } \\
\hline Arachnophaga Ashmead, 1896* & Corumbá \\
\hline Eupelmus Dalman, 1820 & Corumbá \\
\hline \multicolumn{2}{|l|}{ Eurytomidae } \\
\hline Bephrata Cameron, 1884* & Corumbá \\
\hline Eurytoma Illiger, $1807 *$ & Corumbá \\
\hline Neorileya Ashmead, 1904* & Corumbá \\
\hline Prodecatoma Ashmead, 1904* & Corumbá \\
\hline Sycophila Walker, 1871* & Corumbá \\
\hline \multicolumn{2}{|l|}{ Mymaridae } \\
\hline Alaptus Westwood, 1839* & Corumbá \\
\hline Gonatocerus Nees, 1834* & Corumbá \\
\hline Neomymar Crawford, 1913* & Corumbá \\
\hline Omyomymar Schauff, 1983* & Corumbá \\
\hline \multicolumn{2}{|l|}{ Ormyridae* } \\
\hline Ormyrus Förster, 1856* & Corumbá \\
\hline \multicolumn{2}{|l|}{ Perilampidae* } \\
\hline Perilampus Latreille, 1809* & Corumbá \\
\hline \multicolumn{2}{|l|}{ Pteromalidae } \\
\hline Chalcedectus Walker, 1852 & Corumbá \\
\hline Spalangia Latreille, 1805 & Corumbá \\
\hline \multicolumn{2}{|l|}{ Signiphoridae* } \\
\hline Thysanus Walker, 1840* & Campo Grande \\
\hline \multicolumn{2}{|l|}{ Tanaostigmatidae* } \\
\hline Tanaostigmodes Ashmead, 1896* & Corumbá \\
\hline Torymidae & \\
\hline Podagrion Spinola, 1811* & Corumbá \\
\hline Torymus Dalman, 1820 & Corumbá \\
\hline CYNIPOIDEA & \\
\hline Figitidae & \\
\hline
\end{tabular}


Tab. II. Cont.

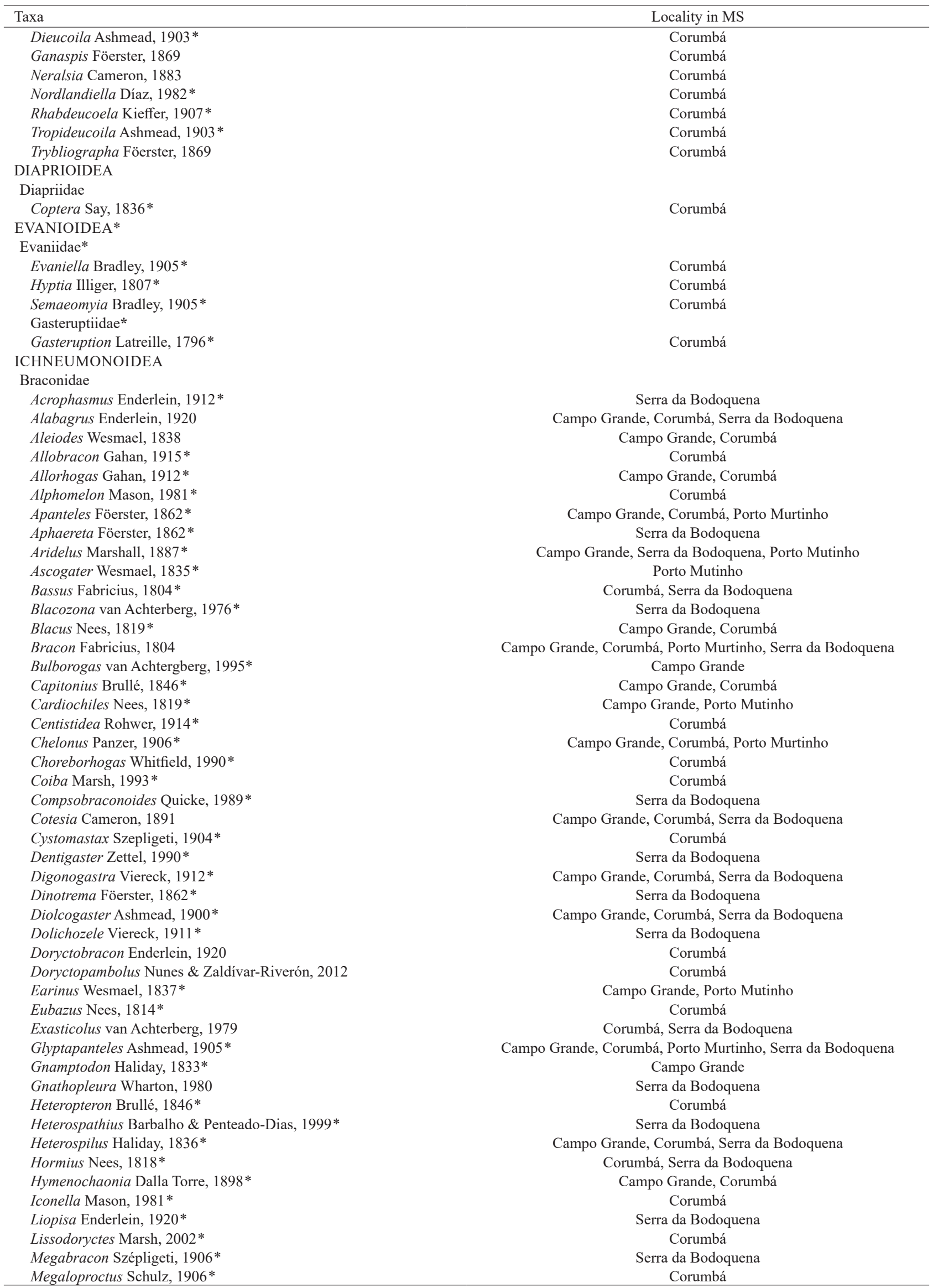


Tab. II. Cont.

\begin{tabular}{|c|c|}
\hline Taxa & Locality in MS \\
\hline Microctonus Wesmael, 1835* & Campo Grande \\
\hline Microplitis Föerster, 1862 * & Campo Grande, Serra da Bodoquena \\
\hline Nealiolus Mason, 1974* & Corumbá, Porto Murtinho \\
\hline Notiospathius Matthews \& Marsh, 1973* & Campo Grande, Serra da Bodoquena \\
\hline Oligoneurus Szépligeti, 1902* & Corumbá \\
\hline Opius Wesmael, 1835 & Campo Grande, Corumbá, Porto Murtinho, Serra da Bodoquena \\
\hline Orgilus Haliday, 1833 & Campo Grande, Corumbá \\
\hline Pambolus Haliday, $1836 *$ & Campo Grande, Corumbá, Serra da Bodoquena \\
\hline Papanteles Mason, $1981 *$ & Porto Murtinho \\
\hline Peristenus Foerster, $1862 *$ & Campo Grande \\
\hline Phaenocarpa Föerster, 1862* & Serra da Bodoquena \\
\hline Phanerotoma Wesmael, 1838* & Campo Grande, Corumbá \\
\hline Pioscelus Muesebeck \& Walkley, 1951* & Campo Grande \\
\hline Platydoryctes Barbalho \& Penteado-Dias, 2000* & Corumbá \\
\hline Promicrogaster Brues \& Richardson, 1913* & Campo Grande \\
\hline Protapanteles Ashmead, 1898* & Serra da Bodoquena, Porto Murtinho \\
\hline Pseudapanteles Ashmead, 1898* & Campo Grande, Corumbá \\
\hline Pseudognamptodon Fischer, 1964* & Corumbá, Serra da Bodoquena \\
\hline Rhaconotus Ruthe, $1854 *$ & Corumbá \\
\hline Rogas Nees, $1818^{*}$ & Campo Grande, Corumbá \\
\hline Semirhytus Szépligeti, 1902* & Serra da Bodoquena \\
\hline Snellenius Westwood, $1882 *$ & Corumbá, Serra da Bodoquena \\
\hline Stantonia Ashmead, 1904* & Corumbá \\
\hline Stiropius Cameron, 1911 & Corumbá, Serra da Bodoquena \\
\hline Streblocera Westwood, 1833* & Serra da Bodoquena \\
\hline Triaspis Haliday, $1835^{*}$ & Corumbá \\
\hline Urosigalphus Ashmead, 1888* & Campo Grande, Corumbá \\
\hline Zelomorpha Ashmead, 1900* & Corumbá, Serra da Bodoquena \\
\hline \multicolumn{2}{|l|}{ Ichneumonidae } \\
\hline Anomalon Panzer, 1804* & Corumbá \\
\hline Brachycyrtus Hoffer, 1957* & Campo Grande \\
\hline Casinaria Holmgren, $1859^{*}$ & Corumbá, Campo Grande \\
\hline Colpotrochia Holmgren, 1855* & Corumbá \\
\hline Dusona Cameron, $1900^{*}$ & Corumbá \\
\hline Eiphosoma Cresson, 1865 & Corumbá, Campo Grande \\
\hline Enicospilus Stephens, $1835^{*}$ & Corumbá, Rio Brilhante \\
\hline Labena Cresson, $1864 *$ & Corumbá \\
\hline Lycorina Holmgren, 1859* & Campo Grande \\
\hline Microcharops Roman, $1910^{*}$ & Corumbá, Campo Grande \\
\hline Neotheronia Krieger, 1898* & Corumbá \\
\hline Netelia Gray, $1860^{*}$ & Corumbá, Campo Grande \\
\hline Ophion Fabricius, 1798* & Rio Brilhante \\
\hline Ophionellus Westwood, 1874* & Campo Grande \\
\hline Pimpla Fabricius, $1804 *$ & Corumbá \\
\hline Podogaster Brullé, 1846* & Corumbá \\
\hline Pristomerus Curtis, $1863 *$ & Corumbá \\
\hline Trieces Townes, 1946* & Corumbá \\
\hline Venturia Schrottky, 1902 & Campo Grande \\
\hline
\end{tabular}

in the North region are INPA, Manaus, (AM) and MPEG, Belém (PA). In the Center-West region holdings of insect collections are found in the following institutions: Reserva Ecológica do IBGE, Brasília (DF); Depto. de Zoologia da UNB, Brasília (DF); EMBRAPA-CPAC, Planaltina (DF); UNEMAT campus Nova Xavantina, Nova Xavantina (MT); UCDB, Campo Grande (MS); UFMS, Campo Grande (MS); UFGD, Dourados (MS).

Main knowledge gaps. As identified by FERNÁNDEZ \& SHARKEY (2006), the Neotropical region lacks studies for Ceraphronoidea, Cynipoidea, Evanioidea, Platygastroidea and Chalcidoidea. This list could be added with the Proctotrupoidea and Diaprioidea, currently with no specialists in Brazil. In despite of the 105 species listed with occurrence in the state, it is possible to notice that according to the list of genera presented here, little is known about the fauna in the region for all groups studied. The large taxonomic knowledge gap is not a particularity of the state. Among the more urgent efforts in need for research of the group, taxonomic revisions of the Neotropical genera are recognized to be the main ones. This is the first step for any other research program concerned with biodiversity, e.g. conservation, evolution and biogeography.

Efforts like the Biota-MS Program are primary to fulfilling this gap through surveys over this region. Considering the regions and vegetal formations, the need for surveys seems more evident in areas of Pantanal, Chaco and on the state's remaining forest fragments. Our results point 
that the best sampled is the Pantanal area that belongs to the Municipality of Corumbá (MS), containing even endemic groups. We believe that there is a need for the integration of local researchers with specialists from the different taxonomic groups in order to establish research lines in that knowledge area.

Research perspectives for the next ten years. By analyzing the publications, it can be observed that since the state's creation a significant increase occurred on the number of works involving this fauna. This increase could be even more significant if the country's main research groups on "Parasitica" and local researchers could reach integration. If the gaps being pointed out here could be filled up during the following years, it is possible that a large number of species could be known and described and the distribution of species already known could be extended throughout the state of Mato Grosso do Sul. With a better knowledge about the species and their distribution, it is possible that this group could become useful as a means for the evaluation of priority areas for environmental conservation, since they include a significant percentage of the biota and show quick response to environmental changes.

Acknowledgements. We would like to thank Fundação de Apoio ao Desenvolvimento do Ensino, Ciências e Tecnologia do Estado de Mato Grosso do Sul (Fundect) e Superintendência de Ciências e Tecnologia do Estado de Mato Grosso do Sul (Sucitec/MS) for the invitation to participate in this special issue of Iheringia, Série Zoologia and financial support for publication. To Dr. Nelson Wanderley Perioto for his help on the identification of the Chalcidoidea. To the students at the Biological Science and Graduate Program in Entomology and Biology Preservation at Universidade Federal da Grande Dourados, for the collection and availability of the material, in particular to Vander Carbonari and Rosália Azambuja. To Dr. Antonia Railda Roel for her encouragement to carry out research on parasitoids in the state of Mato Grosso do Sul and the availability of entomological material. To the fostering institutions that provided the financial support for the realization of this work, namely: Fundação de Amparo à Pesquisa do Estado de São Paulo (FAPESP), Fundação de Apoio ao Desenvolvimento do Ensino, Ciência e Tecnologia do Estado de Mato Grosso do Sul (FUNDECT), Conselho Nacional de Ciência e Tecnologia $(\mathrm{CNPq})$ and Coordenação de Aperfeiçoamento de Pessoal de Nível Superior (CAPES). Finally, to Instituto Nacional de Ciência e Tecnologia dos Hymenoptera Parasitoides da Região Sudeste Brasileira (HYMPAR/Sudeste - CNPq/FAPESP/CAPES).

\section{REFERENCES}

Aguiar, A. P. 1998. Revisão do gênero Hemistephanus Enderlein, 1906 (Hymenoptera: Stephanidae), com considerações metodológicas. Revista Brasileira de Entomologia 41(2-4):343-429.

Aguiar, A. P. 2004. World catalog of the Stephanidae (Hymenoptera: Stephanoidea). Zootaxa 753:1-120.

Aguiar, A. P. \& Ramos, A. C. B. 2011. Revision of Digonocryptus Viereck (Hymenoptera: Ichneumonidae: Cryptinae), with twenty-six new taxa and cladistic interpretation of two species complexes. Zootaxa 2846:1-98.

Andrade, T. V. \& Tavares, M. T. 2009. Revisão de Ceyxia Girault, stat. rev. (Hymenoptera, Chalcididae, Brachymeriini). Revista Brasileira de Entomologia 53(4):511-548.

Andrade Filho, N. N.; Roel, A. R.; Penteado-Dias, A. M. \& Costa, R. B. 2012. Biology of Bemisia tuberculata Bondar (Aleyrodidae) and parasitism by Encarsia porteri (Mercet, 1928) (Hymenoptera, Aphelinidae) on cassava. Brazilian Journal of Biology 72(4):903-907.

ARCE, C. C. M. 2007. Parasitismo natural em ovos de Euschistus heros (Hemiptera: Pentatomidae) na cultura da soja, na região de Dourados, MS. Trabalho de Conclusão de Curso. Dourados, Universidade Federal da Grande Dourados, UFGD. 18p.

Arouca, R. G. \& Penteado-Dias, A. M. 2009. First record of four Alysiinae species (Hymenoptera, Braconidae) from Brazil. Brazilian Journal of Biology 69(4):1209-1210.

Ashmead, W. H. 1904. Classification of the Chalcid Flies or The Superfamily Chalcidoidea with descriptions of New Species in the Carnegie Museum, collected in South America by Herbert H. Smith. Memoirs of the Carnegie Institute 1(4):225-551.

Bellon, P. P.; Favero, K.; Tavares, M. T. \& Oliveira, H. N. 2013. First record of Euplectrus floryae (Hymenoptera: Eulophidae) parasitizing Erinnyis ello (Lepidoptera: Sphingidae) in Brazil. Revista Colombiana de Entomología 39(1):166-167.

Berti-Filho, E.; Mendes-Filho J. M. A. \& Krügner T. L. 1980. Pragas e doenças de Eucalyptus na região do Mato Grosso do Sul. Circular Técnica do Instituto de Pesquisas e Estudos Florestais 106:1-12.

Botelho, P. S. M.; Parra, J. R. P.; Chagas Neto, J. F. \& Oliveira, C. P. B. 1999. Associação do parasitóide de ovos Trichogramma galloi Zucchi (Hymenoptera: Trichogrammatidae) e do parasitóide Larval Cotesia flavipes (Cam.) (Hymenoptera: Braconidae) no controle de Diatraea saccharalis, (Fabr.) (Lepidoptera: Crambidae) em cana-deaçúcar. Anais da Sociedade Entomológica do Brasil 28(3):491-496.

Braet, Y. \& van AChterberG, C. 2001. New taxa of the subfamily Doryctinae Foerster (Hymenoptera: Braconidae) from French Guiana and Brazil. Zoologische Mededelingen 75(7):119-136.

Brandão, C. R. F.; Cancello, E. M. \& Yamamoto, C. I. 2000. Avaliação do estado atual do conhecimento sobre a diversidade biológica de invertebrados terrestres no Brasil. Relatório final. In: LEwINSOHN, T. ed. Avaliação do estado do conhecimento da diversidade biológica do Brasil. Brasília, MMA - GTB/CNPq - NEPAM/UNICAMP, p.141-147.

Caires, C. S.; UchôA-Fernandes, M. A.; NicÁcio, J. \& Strikis, P. C. 2009. Frugivoria de larvas de Neosilba McAlpine (Diptera, Lonchaeidae) sobre Psittacanthus plagiophyllus Eichler (Santalales, Loranthaceae) no sudoeste de Mato Grosso do Sul, Brasil. Revista Brasileira de Entomologia 53(2):272-277.

CAMPos, A. R.; LaRA, F. M. \& CAMPOS, O. R. 1998. Influence of sorghum genotype on the sorghum midge, Stenodiplosis sorghicola (Diptera: Cecidomyiidae) and its parasitoid Aprostocetus diplosidis Crawford, 1907 (Hymenoptera, Eulophidae). Revista Cultura Agronômica 7(1):91-100.

CARBONARI, V. 2009. Composição faunística de vespas (Hymenoptera: Apocrita) do Parque Nacional da Serra da Bodoquena. Dissertação de Mestrado. Dourados, Universidade Federal da Grande Dourados. 56p.

Carvalho, E. S. M. 2007. Dichelops melacanthus (Dallas, 1851) (Heteroptera: Pentatomidae) no sistema plantio direto no sul de Mato Grosso do Sul: flutuação populacional, hospedeiros e parasitismo. Dissertação de Mestrado em Entomologia e Conservação da Biodiversidade. Dourados, Universidade Federal da Grande Dourados. 41p.

COMPERE, H. 1937. The species of Aenasius, encyrtid parasites of mealybugs. Proceedings of the Hawaiian Entomological Society 9:383-404.

Costa Lima, A. 1953. Espécies de Eiphosoma Cresson (Hymenoptera, Ichneumonidae, Ophioninae). Arquivos do Museu Nacional 42(1951):175-189.

De SANTIS, L. 1980. Catalogo de los Himenopteros Brasileños de la serie Parasitica incluyendo Bethyloidea. Curitiba, Editora da Universidade Federal do Paraná. 395p.

Delvare, G. 1992. A reclassification of the Chalcidini with a check list of the New World species. In: Delvare, G. \& BoučeK, Z. eds. On the New World Chalcididae (Hymenoptera). Memoirs of the American Entomological Institute 53:119-467.

Díaz, N. B. \& Gallardo, F. 1996. Sobre cinipoideos del Brasil, parasitoides de dipteros estercoleros (Hymenoptera: Cynipoidea). Revista de la Sociedad Entomológica Argentina 55(1-4):127-129.

Díaz, N. B.; Gallardo, F. E.; Marchiori, C. H. \& Linhares, A. X. 2000. Cynipoidea parasitoids of dung-flies in Brazil. II (Insecta: Hymenoptera). Anais da Sociedade Entomológica do Brasil 29(3):469-474.

Fernandes, M. G.; Bussoli, A. C. \& Degrande, P. E. 1999. Parasitismo natural de ovos de Alabama argillacea Hüb. e Heliothis virescens Fab. (Lep.: Noctuidae) por Trichogramma pretiosum Riley (Hym.: Trichogrammatidae) em algodoeiros no Mato Grosso do Sul. Anais da Sociedade Entomológica do Brasil 28(4):695-701. 
Fernández, F. 2006. Sistemática de los himenópteros de la Región Neotropical. Estado del conocimiento y perspectivas. In: FERnÁNDEZ, F. \& Sharkey, M. J. eds. Introducción a los Hymenoptera de la Región Neotropical. Bogotá, Sociedad Colombiana de Entomología, Universidad Nacional de Colombia, p.7-36. (Serie Entomología Colombiana).

FERnÁNDEZ, F. \& SHARKeY, M. J. 2006. Introducción a los Hymenoptera de la Región Neotropical. Bogotá, Sociedad Colombiana de Entomología, Universidad Nacional de Colombia. 896p. (Serie Entomología Colombiana).

Figueiredo, M. L. C.; Penteado-Dias, A. M. \& Cruz, I. 2006. Exasticolus fuscicornis em lagartas de Spodoptera frugiperda. Pesquisa Agropecuária Brasileira 41(8):1321-1323.

Gallardo, F. E.; Díaz, N. B. \& UchôA-Fernandes, M. A. 2000. A new species of Trybliographa (Hymenoptera: Figitidae: Eucoilinae) from Brazil associated with fruit infesting Lonchaeidae (Diptera). Revista de la Sociedad Entomológica Argentina 59:21-24

Gaston, K. J. 1991. The magnitude of global insect species richness. Conservation Biology 5:283-296.

Gaston, K. J.; Gauld, I. D. \& Hanson, P. 1996. The size and composition of the hymenopteran fauna of Costa Rica. Journal of Biogeography 23:105-113.

Gibson, G. A. P. 2009. Revision of New World Spalangiinae (Hymenoptera: Pteromalidae). Zootaxa 2259:1-159.

Godoy, K. B. \& Ávila, C. J. 2000. Parasitismo natural em ovos de dois percevejos da soja, na região de Dourados, MS. Revista de Agricultura 75(2):271-279.

Godoy, K. B.; Ávila, C. J. \& Arce, C. C. M. 2007. Controle Biológico de Percevejos Fitófagos da Soja na Região de Dourados, MS. Dourados, Embrapa Agropecuária Oeste. 27p.

Godoy, K. B.; Ávila, C. J.; Duarte, M. M. \& Arce, C. C. M. 2010. Parasitismo e sítios de diapausa de adultos do percevejo marrom, Euschistus heros na região da Grande Dourados, MS. Ciência Rural 40(5):1199-1202.

Godoy, K. B.; Galli, J. C. \& Ávila, C. J. 2005. Parasitismo em ovos de percevejos da soja Euschistus heros (Fabricius) e Piezodorus guildinit (Westwood) (Hemiptera: Pentatomidae) em São Gabriel do Oeste, MS. Ciência Rural 35(2):455-458.

Gomez, S. A. \& Rumiatto, M. 1989. Criação e liberação de inimigos naturais para o controle de afídeos. Reunião da Comissão Centro-Sulbrasileira de pesquisa do trigo. Resultados da pesquisa com trigo-1988. UEPAED - EMBRAPA Agropecuária Oeste 39:165-166.

Gomez, S. A.; Rohden, V. S.; Duarte, M. M. \& Arce, C. C. M. 2006. Entomofauna Associada à Cultura da Mandioca na Região Sul de Mato Grosso do Sul. Dourados, Embrapa Agropecuária Oeste. 27p.

Grimaldi, D. \& Engel, M. S. 2005. Evolution of the insects. Cambridge, Cambridge University Press. 755p.

Grissell, E. E. 1999. Hymenopteran biodiversity: Some alien notions. American Entomologist 45:235-244.

Guimarẽes, J. A.; Zucchi, R. A.; Díaz, N. B.; Souza Filho, M. F. \& UChÔA-FERnANDES, M. A. 1999. Espécies de Eucoilinae (Hymenoptera: Cynipoidea: Figitidae) parasitóides de larvas frugívoras (Diptera: Tephritidae e Lonchaeidae) no Brasil. Anais da Sociedade Entomológica do Brasil 28(2):263-273.

Johnson, N. F. 1992. Catalog of world species of Proctotrupoidea, exclusive of Platygastridae (Hymenoptera). Memoirs of the American Entomological Institute 51:1-825.

Kunzler, J.; Fernandes, A. C. S.; Fonseca, V. M. M. \& Jraige, S. 2011. Herbert Huntington Smith: um naturalista injustiçado? Filosofia e História da Biologia 6(1):49-67.

Lewinsohn, T. M.; Freitas, A. V. L. \& Prado, P. I. 2005. Conservation of terrestrial invertebrates and their habitats in Brazil. Conservation Biology 19(3):640-645.

Lewinsohn, T. M. \& Prado, P. I. 2002. Biodiversidade Brasileira. Síntese do estado atual do conhecimento. São Paulo, Contexto; Ministério do Meio Ambiente e Conservation International do Brasil. 176p.

Marsh, P. M. 1983. Bohartiellus, a new genus of Doryctinae from South America (Hymenoptera: Braconidae). Pan-Pacific Entomologist 59:138-151.

Marsh, P. M. 1993. Descriptions of new Western Hemisphere genera of the subfamily Doryctinae (Hymenoptera: Braconidae). Contributions of the American Entomological Institute 28(1):1-58.

Mercet, R. G. 1926. Los géneros Chalcaspis How. y Eugahania nuevo (Hym. Chalc.). Eos 2:43-48.

Mussury, R. M. \& Fernandes, W. D. 2002. Occurrence of Diaretiella rapae (Mc'Intosh, 1855) (Hymenoptera: Aphidiidae) parasitising Lipaphis erysimi (Kaltenbach, 1843) and Brevicoryne brassicae (L., 1758) (Homoptera: Aphididae) in Brassica napus in Mato Grosso do Sul. Brazilian Archives of Biology and Technology 45:41-46.

Noyes, J. S. 2013. Universal Chalcidoidea Database. World Wide Web electronic publication. Available at: <http://www.nhm.ac.uk/ chalcidoids $>$.

Nunes, J. F.; Zaldívar-Riverón, A.; Castro, C. S.; Marsh, P. M.; PenteadoDias, A. M.; Briceño, R. \& MartíneZ, J. J. 2012. Doryctopambolus Nunes \& Zaldívar-Riverón (Braconidae), a new neotropical doryctine wasp genus with propodeal spines. Zookeys 223:53-67.

Oliveira, H. N. \& Silva, C. J. 2011. Artrópodes benéficos na cultura do Pinhão-Manso em Mato Grosso do Sul. Comunicado Técnico 164:1-4.

Oliveira, H. N.; Gomez, S. A.; Rohden, V. S.; Arce, C. C. M. \& Duarte, M. M. 2010. Record of Trichogramma (Hymenoptera: Trichogrammatidae) species on Erinnyis ello Linnaeus (Lepidoptera: Sphingidae) eggs in Mato Grosso do Sul State, Brazil. Pesquisa Agropecuária Tropical 40(3):378-379.

Richards, O. W. 1978. The Social Wasps of Americas excluding the Vespinae. London, British Museum (Natural History). 580p.

Sereno, F. T. P. S. \& Sereno, J. R. B. 2000. Parasitismo em pupas de Haematobia irritans por parasitóides nativos do pantanal. Simpósio sobre recursos naturais e sócio-econômicos do pantanal - Os desafios do novo milênio. Corumbá, MS.

Sereno, F. T. P. S. 2000. Pupas de mosca-dos-chifres, Haematobia irritans, em massas fecais de bovinos nelore no pantanal. Pesquisa Agropecuária Brasileira 35(8):1685-1688.

Sharkey, M. J. 1986. Pharpa, a new genus of Neotropical Agathidinae (Hymenoptera: Braconidae) with a discussion of phylogenetic relationships. Canadian Entomologist 118:1231-1239.

SHARKeY, M. J. 1988. A taxonomic revision of Alabagrus (Hymenoptera: Braconidae). Bulletin of the British Museum (Natural History), Entomology 57:311-437.

Sharkey, M. J. 2007. Phylogeny and Classification of Hymenoptera. Zootaxa 1668:521-548.

Sharkey, M. J., Carpenter, J. M., Vilhemsen, L., Heraty, J., Liljeblad, J., Dowling, A. P. G., Schulmeister, S., Murray D., Deans, A. R., Ronquist, F., Krogmannu, L. \& Wheeler W. C. 2012. Phylogenetic relationships among superfamilies of Hymenoptera. Cladistics 28:80112.

Shenefelt, R. D. 1969. Braconidae 1, Hybrizoninae, Euphorinae, Cosmophorinae, Neoneurinae, Macrocentrinae. In: VeCHT, J. V. D. \& Shenefelt, R. D. eds. Hymenopterorum Catalogus (new edition). The Hague, Junk. Pars 4, p.1-176.

Shenefelt, R. D. 1970a. Braconidae 2, Helconinae, Calyptinae, Mimagathidinae, Triaspidinae. In: Vecht, J. V. D. \& Shenefelt, R. D. (eds). Hymenopterorum Catalogus (new edition). Junk, The Hague. Pars 5, p.177-306.

Shenefelt, R. D. 1970b. Braconidae 3, Agathidinae. In: Vecht, J. V. D. \& Shenefelt, R. D. eds. Hymenopterorum Catalogus (new edition). The Hague, Junk. Pars 6, p.307-428.

Shenefelt, R. D. 1972. Braconidae 4, Microgasterinae, Apanteles. In: Vecht, J. V. D. \& Shenefelt, R. D. eds. Hymenopterorum Catalogus (new edition). Junk, The Hague. Pars 7, p.429-668.

Shenefelt, R. D. 1973a. Braconidae 5, Microgasterinae, Ichneutinae. In: Vecht, J. V. D. \& Shenefelt, R. D. eds. Hymenopterorum Catalogus (new edition). Junk, The Hague. Pars 9, p.669-812.

Shenefelt, R. D. 1973b. Braconidae 6, Cheloninae. In: Vecht, J. V. D. \& Shenefelt, R. D. eds. Hymenopterorum Catalogus (new edition). The Hague, Junk. Pars 10, p. 813-936.

Shenefelt, R. D. 1974. Braconidae 7, Alysiinae. In: Vecht, J. V. D. \& Shenefelt, R. D. eds. Hymenopterorum Catalogus (new edition). The Hague, Junk. Pars 11, p.937-1113.

_Shenefelt, R. D. 1975. Braconidae 8, Exothecinae, Rogadinae. In: Vecht, J. V. D. \& Shenefelt, R. D. eds. Hymenopterorum Catalogus (new edition). Junk, The Hague. Pars 12, p. 1115-1262. 
Shenefelt, R. D. 1978. Braconidae 10, Braconinae, Gnathobraconinae, Mesostoinae, Pseudodicrogeniinae, Telengainae, Ypsistocerinae plus Braconidae in general, major groups, unplaced genera and species. In: van AchterberG, C. \& Shenefelt, R. D. eds. Hymenopterorum Catalogus (new edition). The Hague, Junk. Pars 15, p.1425-1872.

Shenefelt, R. D. 1980. Braconidae 11, Introduction, Guide to host names, index to braconid names. In: van AchterberG, C. \& Shenefelt, R. D. eds. Hymenopterorum Catalogus (new edition). The Hague, Junk. Pars 16, p.1-384.

Shimbori, E. M. \& Penteado-Dias, A. M. 2011. Taxonomic contribution to the Aleiodes melanopterus (Erichson) species-group (Hymenoptera, Braconidae, Rogadinae) from Brazil. ZooKeys 142:15-25.

Silveira, N. A.; Madeira, N. G.; Azeredo-Espin, A. M. L. \& Pavan, C. 1989. Levantamento de microhimenópteros parasitóides de dípteros de importância médico-veterinárioa no Brasil. Memórias do Instituto Oswaldo Cruz 84(4):505-510.

Sмith, D. R. 2001. World catalog of the family Aulacidae (Hymenoptera). Contributions on Entomology International 4(3):263-319.

Smith, H. H. 1922. Do Rio de Janeiro a Cuyabá: notas de um naturalista por Herbert H. Smith. Com um capitulo de Carlos von den Steinen sobre a capital de Matto Grosso. Cayeiras, Companhia Melhoramentos de São Paulo. 376p.

Starý, P.; Sampaio, M. V. \& Bueno, V. H. P. 2007. Aphid parasitoids (Hymenoptera, Braconidae, Aphidiinae) and their associations related to biological control in Brazil. Revista Brasileira de Entomologia 51(1):107-118.

Stork, N. E. 1991. The composition of the arthropod fauna of Bornean lowland rainforest trees. Journal of Tropical Ecology 7:161-180.

Townes, H. \& TownEs M. 1966. A catalogue and reclassification of neotropic Ichneumonidae. Memoirs of the American Entomological Institute 8:1-367.

Uchôa-Fernandes, M. A.; Molina, R. M. S.; Oliveira, I.; Zucchi, R. A.; CANAL, N. A. \& DíAz, N. B. 2003a. Larval endoparasitoids (Hymenoptera) of frugivorous flies (Diptera, Tephritoidea) reared from fruits of the cerrado of the State of Mato Grosso do Sul, Brazil Revista Brasileira de Entomologia 47(2):181-186

UchôA-Fernandes, M. A.; Ollveira, I.; Molina, R. M. S. \& Zucchi, R. A. 2003b. Populational fluctuation of frugivorous flies (Diptera: Tephritoidea) in two orange groves in the state of Mato Grosso do Sul, Brazil. Neotropical Entomology 32(1):19-25.

VALÉRIO, J. R. \& OliveIRA, M. C. M. 2005. Parasitismo de ovos de cigarrinhasdas-pastagens (Homoptera: Cercopidae) pelo microhimenóptero Anagrus urichi Pickles (Hymenoptera: Mymaridae) na Região de Campo Grande, MS. Neotropical Entomology 34(1):137-138.

Vargas E. L.; Pereira F. F.; Tavares M. T. \& Pastori P. L. 2011. Record of Tetrastichus howardi (Hymenoptera: Eulophidae) parasitizing Diatraea sp. (Lepidoptera: Crambidae) in sugarcane crop in Brazil. Entomotropica 26(3):143-146.

VLuG, H. J. 1995. Catalogue of the Platygastridae (Platygastroidea) of the World. In: van ACHTERBERG, C. ed. Hymenopterorum Catalogus (new edition). Amsterdam, SBP Academic Publishing. Pars 19, 168p.

Weinstein, P. \& Austin, A. D. 1991. The host relationships of trigonalyid wasps (Hymenoptera: Trigonalyidae), with a review of their biology and catalogue to world species. Journal of Natural History 25:399-433.

Whitfield, J. B. 1992. The polyphyletic origin of endoparasitism in the cyclostome lineages of Braconidae (Hymenoptera). Systematic Entomology 17:273-286.

Whitfield, J. B. 2003. Phylogenetic insights into the evolution of parasitism in Hymenoptera. Advances in Parasitology 54:69-100.

Yu, D. S.; van Achterberg, C. \& Horstmann, K. 2012. World Ichneumonoidea 2012. Taxonomy, biology and distribution. Ottawa, Taxapad (DVD/CD-ROOM).

Zaldívar-Riverón, A.; Belokobilskij, S. A.; León-Regagnon, V.; Martinez, J. J.; Briseño, R. \& Quicke, D. L. J. 2007. A single origin of gall association in a group of parasitic wasps with disparate morphologies. Molecular Phylogenetics and Evolution 44:981-992.

Zucchi, R. A. \& Monteiro, R. C. 1995. New records of hosts and distribution of Trichogramma species in Brazil. Les Colloques L'INRA 73:51-53 